\title{
Normalized Structured Compressed Sensing Based Signal Detection in Spatial Modulation 3D-MIMO Systems
}

\author{
Wei Ren, Guan Gui, and Fei Li \\ College of Telecommunication and Information Engineering, Nanjing University of Posts and Telecommunications, \\ Nanjing 210003, China \\ Correspondence should be addressed to Guan Gui; guiguan@njupt.edu.cn
}

Received 15 September 2017; Revised 5 November 2017; Accepted 15 November 2017; Published 6 December 2017

Academic Editor: Nan Zhao

Copyright (C) 2017 Wei Ren et al. This is an open access article distributed under the Creative Commons Attribution License, which permits unrestricted use, distribution, and reproduction in any medium, provided the original work is properly cited.

\begin{abstract}
Signal detection is one of the fundamental problems in three-dimensional multiple-input multiple-output (3D-MIMO) wireless communication systems. This paper addresses a signal detection problem in 3D-MIMO system, in which spatial modulation (SM) transmission scheme is considered due to its advantages of low complexity and high-energy efficiency. SM based signal transmission typically results in the block-sparse structure in received signals. Hence, structured compressed sensing (SCS) based signal detection is proposed to exploit the inherent block sparsity information in the received signal for the uplink (UL). Moreover, normalization preprocessing is considered before iteration process with the purpose of preventing the noise from being overamplified by the column vector with inadequately large elements. Simulation results are provided to show the stable and reliable performance of the proposed algorithm under both Gaussian and non-Gaussian noise, in comparison with methods such as compressed sensing based detectors, minimum mean square error (MMSE), and zero forcing (ZF).
\end{abstract}

\section{Introduction}

Multiple-input multiple-output (MIMO) systems improve reliability and spectral efficiency of communication systems as a result of multiple antennas and have been included in the fourth generation $(4 \mathrm{G})$ mobile communication standards $[1,2]$. Nowadays, three-dimensional multiple-input multipleoutput (3D-MIMO) system, equipped in a 2D planar array with a large number of active antennas, has shown large potential in increasing system capacity [3-5]. In 3rd Generation Partnership Project (3GPP) Release 12, 3D-MIMO system has been considered as an important technique for throughput enhancement and multiuser interference mitigation $[6,7]$. Signal detection is one of the fundamental problems in 3D-MIMO wireless communication systems due to the requirement of synchronization between the antennas and the strong interchannel interference (ICI) between each of the receive antennas [8].

Spatial modulation (SM) has attracted much research attention as a novel multiantenna transmission scheme due to its advantages of low complexity and high-energy efficiency $[9,10]$. In addition to phase and amplitude modulation, SM can also utilize transmit antennas' indices as the third dimension in order to invoke for more transmitting information. Unlike the traditional MIMO systems, the SM transmitter in 3D-MIMO systems uses massive transmit antennas but a small number of radio frequency (RF) chains, which significantly improves energy efficiency of the whole system, because the hardware cost and power consumption increase highly with the number of RF chains [10,11]. With only one or several transmit antennas being active at each time slot, ICI at the receiver and the need to synchronize the transmit antennas can be largely reduced [12-15]. Moreover, with only one or several nonzero components in transmit signal at each slot, the inherent sparsity of SM-3D-MIMO signals can be utilized in signal detection. Since users have the same active antenna selection scheme and share the same spatial constellation symbol, the received signal has the block-sparse structure which can be utilized in signal detection via structure compressed sensing.

For the novel transmit systems, suitable signal detection algorithms are required to obtain signals. The maximumlikelihood (ML) detector has too high complexity which will 


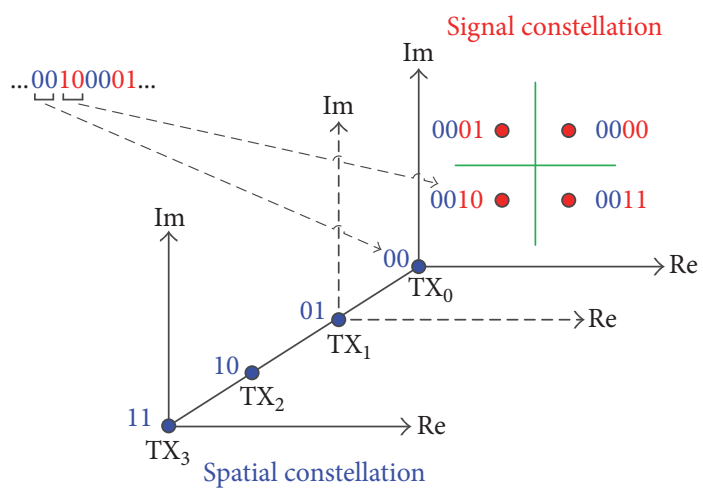

FIGURE 1: Signal constellation symbol and spatial constellation symbol in the SM 3D-MIMO system, where $N_{t}=4, N_{a}=1$, and 4QAM are taken into account as an example.

linearly increase with the size of the symbol constellation and the numbers of transmit and receive antennas [16]. Sphere decoding (SD) based [17] and linear minimum mean square error (LMMSE) based signal detecting method will suffer from large and considerable performance reduction in the SM-MIMO systems [18]. Compressed sensing (CS) theory should be used to reliably improve the signal detecting performance due to the exploiting of the inherent sparsity information in SM signals [19, 20]. In [21], CS theory is used for signal detection in multiple access large-scale channels. In [22], a structured compressed sensing based signal detecting algorithm is proposed for the massive spatial modulation MIMO systems. Paper [23] gives a performance evaluation of the existing signal detecting algorithms such as SCS based SSP algorithm, CS based SP algorithm, and other conventional methods. On the basis of existing near-optimal signal detection algorithms, this paper proposes a modified SCS based algorithm for the spatial modulation 3D-MIMO systems.

The rest of this paper is organized as follows. Section 2 presents spatial modulation, maximum-likelihood detection, and compressed sensing. Section 3 introduces the proposed normalized structured subspace pursuit algorithm. Section 4 gives simulation results and performance analysis of several signal detectors.

\section{System Model}

Spatial Modulation. In spatial modulation MIMO systems, the transmitter has $N_{t}$ transmit antennas and $N_{a}<N_{t}$ active antennas, and the receiver has $N_{r}$ receive antennas. The information bit stream is grouped into two parts: the first part with $\left\lfloor\log _{2}\left(\begin{array}{c}N_{t} \\ N_{a}\end{array}\right)\right\rfloor$ bits is mapped onto the spatial constellation symbols which indicate different selection schemes of the active transmit antennas, and the second part with $\log _{2} L$ bits is mapped onto the signal constellation symbols derived from the signal constellation set of $L$-ary. Therefore, the total information carried by each SM signal is $N_{a} \log _{2} L+\left\lfloor\log _{2}\left(\begin{array}{c}N_{t} \\ N_{a}\end{array}\right)\right\rfloor$ bits. Figure 1 shows an example of signal constellation symbol and spatial constellation symbol in the spatial modulation 3D-MIMO system.

At the receiver, the received signal $\mathrm{y} \in \mathbb{C}^{N_{r} \times 1}$ is equal to

$$
\mathbf{y}=\mathbf{H x}+\mathbf{w}
$$

where $\mathbf{x} \in \mathbb{C}^{N_{t} \times 1}$ is the SM signal generated from the transmitter and $\mathbf{w} \in \mathbb{C}^{N_{r} \times 1}$ is the additive noise vector. $\mathbf{H}=$ $\mathbf{R}_{r}^{1 / 2} \widetilde{\mathbf{H}} \mathbf{R}_{t}^{1 / 2} \in \mathbb{C}^{N_{r} \times N_{t}}$ represents the correlated flat Rayleighfading MIMO channel, and $\widetilde{\mathbf{H}}$ is composed of the entries which obey the independent and identical distribution $\mathscr{C} \mathcal{N}(0,1) . \mathbf{R}_{r}$ is the correlation matric of the receiver and $\mathbf{R}_{t}$ is the correlation matric of the transmitter [24]. $\mathbf{R}$ is the correlation matrix composed of $r_{i j}=r^{|i-j|}$ and $r$ is the correlation coefficient between neighboring antennas. Obviously, it is a nondeterministic polynomial hard (NP-hard) detection problem at the receiver.

Maximum-Likelihood (ML) Detection. ML signal detection is the optimal signal detector to the NP-hard problem [25]. It can be expressed as

$$
\widehat{\mathbf{x}}_{\mathrm{ML}=\arg } \min _{\operatorname{supp}(\mathbf{x}) \in \mathbb{A}, \mathbf{x} \in \mathbb{B}}\|\mathbf{y}-\mathbf{H x}\|_{2} .
$$

However, the ML signal detector is unrealistic with the high computational complexity of $\left.\mathcal{O}\left(M^{N_{a}} 2 \log _{2}\left(\begin{array}{c}N_{t} \\ N_{a}\end{array}\right)\right\rfloor\right)$ when $N_{t}, N_{a}$, and $M$ are large $[16,26]$.

Compressed Sensing (CS). Using sparsity, compressed sensing can provide reliable detection for $s$-sparse signals. CS is formulated as $\mathbf{y}=\boldsymbol{\Phi} \mathbf{x}$, where $\mathbf{y} \in \mathbb{R}^{m}$ is the measurement vector and $\Phi \in \mathbb{R}^{m \times n}$ is the measurement matrix with $m<$ $n$. Moreover, $\Phi$ is a matrix satisfying the restricted isometry property (RIP) $[27,28], m$ is greater than $c_{1} s \log (n / s)$ for some small constant $c_{1}$, and $\mathbf{x}$ can be reconstructed by $\ell_{1}$ minimization as

$$
\widehat{\mathbf{x}}=\arg \min _{\mathbf{y}=\boldsymbol{\Phi} \boldsymbol{x}}\|\mathbf{x}\|_{\ell_{1}}
$$

Note that RIP identifies the so-called isometry constant $\delta_{s}$ of the measurement matrix $\Phi$ as the smallest number such that $\left(1-\delta_{s}\right)\|\mathbf{x}\|_{2}^{2} \leq\|\boldsymbol{\Phi} \mathbf{x}\|_{2}^{2} \leq\left(1+\delta_{s}\right)\|\mathbf{x}\|_{2}^{2}$ holds for $s$-sparse vector $\mathbf{x}$. The perfect reconstruction of $s$-sparse vector can be achieved if the sampling matrix $\boldsymbol{\Phi}$ is properly designed to satisfy $\delta_{c_{2} s}<\theta$ for some constant $c_{2}[26,29]$.

\section{The Proposed Signal Detection Algorithm in SM 3D-MIMO Systems}

3.1. Grouped Transmission Scheme. The SM signal $\mathbf{x}_{k}$ is transmitted from the $k$ th user in one time slot and is expressed as $\mathbf{x}_{k}=\mathbf{e}_{k} \mathbf{s}_{k}$. It is composed of two parts: the first part of spatial constellation symbol $\mathbf{e}_{k} \in \mathbb{C}^{n_{t}}$ and the second part of signal constellation symbol $s_{k} \in \mathbb{L}$ which is generated by $L$-ary modulation. Because each user employs only one RF chain, there is just one nonzero entry of $\mathbf{e}_{k}$ which is on behalf 
of the active transmit antenna. Hence, the rest of the entries in $\mathbf{e}_{k}$ are all equal to zero; that is,

$$
\begin{array}{r}
\operatorname{supp}\left(\mathbf{e}_{k}\right) \in \mathbb{A}, \\
\left\|\mathbf{e}_{k}\right\|_{0}=1, \\
\left\|\mathbf{e}_{k}\right\|_{2}=1,
\end{array}
$$

where $\mathbb{A}=\left\{1,2, \ldots, n_{t}\right\}$ represents the spatial constellation symbol set. Therefore, every SM signal $\mathbf{x}_{k}$ contains $\log _{2}(L)+$ $\log _{2}\left(n_{t}\right)$ bits per channel use (bpcu), so the entire throughput coming from the transmitter is $K\left(\log _{2}(L)+\log _{2}\left(n_{t}\right)\right)$ bpcu.

At the transmitter, it is set that one group contains $G$ consecutive SM signals. The SM signals form one group have the same spatial constellation symbol and active antenna selection scheme; that is,

$$
\operatorname{supp}\left(\mathbf{x}_{k}^{1}\right)=\operatorname{supp}\left(\mathbf{x}_{k}^{2}\right)=\cdots=\operatorname{supp}\left(\mathbf{x}_{k}^{G}\right),
$$

where $\mathbf{x}_{k}^{1}, \mathbf{x}_{k}^{2}, \ldots, \mathbf{x}_{k}^{G}$ are SM signals of the $k$ th user in $G$ consecutive symbol slots. Thus they show the feature of structured sparsity, which can be exploited as a priori information to improve the performance of the signal detection.

At the receiver, only $M_{\mathrm{RF}}$ receive antennas are needed to receive signals because of the reductive numbers of RF chains at the BS. With $K$ users concurrently served at the BS, the received signal $\mathbf{y}_{q} \in \mathbb{C}^{M_{\mathrm{RF}}}$ which is form the $q$ th time slot can be expressed as

$$
\mathbf{y}_{q}=\sum_{k=1}^{K} \mathbf{y}_{k, q}+\mathbf{w}_{q}=\sum_{k=1}^{K} \mathbf{H}_{k} \mathbf{x}_{k}+\mathbf{w}_{q},
$$

where $\mathbf{H}_{k} \in \mathbb{C}^{M \times n_{t}}$ represents the MIMO channel matrix from the $k$ th user.

3.2. Normalized Structured Subspace Pursuit Algorithm. The proposed NSSP algorithm is described in Algorithm 1. In the NSSP algorithm, there is normalization preprocessing before the iteration process, after which (1) can be written as

$$
\mathbf{y}=\mathbf{H}^{\prime} \mathbf{C x}+\mathbf{w},
$$

where $\mathbf{C} \in \mathbb{R}^{N_{t} \times N_{t}}$ is a diagonal matrix and its diagonal elements are the $\ell_{2}$-norm of $\mathbf{H}_{-, i}$ denoting the $i$ th column of $\mathbf{H}$. $\mathbf{H}^{\prime}$ is of dimension $N_{r} \times N_{t}$. Let $\mathbf{x}^{\prime}=\mathbf{C x} ;$ (8) can be written as

$$
\mathbf{y}=\mathbf{H}^{\prime} \mathbf{x}^{\prime}+\mathbf{w}
$$

Different from conventional compressed sensing based detectors, where the sensing and recovery processes share the same measurement matrix, the normalization preprocessing allows different matrices during the two processes. After the normalization preprocessing, the noise will not be overamplified by the column vector with inadequately large elements [26].

On the other hand, the spatial constellation set will be exploited as a priori information in the NSSP algorithm. It means that the estimated support set during each iteration

$$
\begin{aligned}
& \text { Input: } \mathbf{y}^{(t)} \text { : the received signal } \\
& \mathbf{H}^{(t)} \text { : the channel matrix } \\
& N_{a} \text { : the number of active antennas } \\
& \text { Output: } \widehat{\mathbf{x}}^{(t)} \text { : the recovery signal for } 1 \leq t \leq G \\
& \text { (1) for } s=1 \text { to } N_{a} \\
& \text { (2) } \quad \text { let } \mathbf{H}_{-, s}^{\prime(t)} \text { be normalized sth column } \mathbf{H}_{-, s}^{(t)} \text { of } \mathbf{H}^{(t)} \\
& \text { (3) } \quad \mathbf{H}^{\prime(t)}=\left[\mathbf{H}_{-, 1}^{\prime(t)} \cdots \mathbf{H}_{-, N_{a}}^{\prime(t)}\right. \\
& \text { (4) } k=1, T_{0}=\varphi, \mathbf{r}^{(t)}=\mathbf{y}^{(t)} \\
& \text { (5) for } k \leq N_{a} \\
& \text { (6) } \quad \mathbf{u}^{(t)}=\left(\mathbf{H}^{\prime(t)}\right)^{*} \mathbf{r}^{(t)} \\
& \text { (7) } \quad P=\arg \max _{P^{\prime}}\left\{\sum_{t=1}^{G}\left\|\mathbf{u}_{P^{\prime}}^{(t)}\right\|_{2}^{2}, P^{\prime} \in \mathbb{A}\right\} \\
& \text { (8) } \quad \Omega=T_{k-1} \cup P^{(t)} \\
& \text { (9) } \quad \mathbf{w}^{(t)}=\left(\mathbf{H}_{\Omega}^{\prime(t)}\right)^{\dagger} \mathbf{y}^{(t)} \\
& \text { (10) } T_{k}=\arg \max _{T^{\prime}}\left\{\sum_{t=1}^{G}\left\|\mathbf{w}_{T^{\prime}}^{(t)}\right\|_{2}^{2}, T^{\prime} \in \mathbb{A}\right\} \\
& \text { (11) } \quad \mathbf{r}^{(t)}=\mathbf{y}^{(t)}-\mathbf{H}^{\prime(t)}\left(\mathbf{H}_{T_{k}}^{\prime(t)}\right)^{\dagger} \mathbf{y}^{(t)} \\
& \text { (12) } \quad k=k+1 \\
& \text { (13) } \widehat{\mathbf{x}}^{(t)}=\left(\mathbf{H}_{T_{k}}^{(t)}\right)^{\dagger} \mathbf{y}^{(t)}
\end{aligned}
$$

Algorithm 1: Our proposed NSSP based signal detection algorithm in spatial modulation 3D-MIMO systems.

should belong to the predefined spatial constellation set. During each iteration, the potential true indices will be obtained according to the correlation between the MIMO channels and the residual in the previous iteration, and then the estimated support set will be updated after the least squares.

It is proved that the recovery performance of SCS based signal detectors is better than the traditional CS based signal detectors with the measurement vectors of the same size [30]. The structured compressed sensing based algorithm can solve multiple sparse signals with common support set but different measurement matrices [22].

The description of the NSSP algorithm is given as follows:

(1) The parameters of input are the measurement vector $\mathbf{y}$, the number of active antennas $N_{a}$, and the channel matrix $\mathbf{H}$.

(2) The normalization preprocessing performs before iteration process. $\mathbf{H}^{\prime}$ is of dimension $N_{r} \times N_{t}$ and $\mathbf{C} \epsilon$ $\mathbb{R}^{N_{t} \times N_{t}}$ is a diagonal matrix whose diagonal elements are the $\ell_{2}$-norm of $\mathbf{H}_{-, i}$ denoting the $i$ th column of $\mathbf{H}$.

(3) In the support merging section, according to the correlation $\mathbf{u}^{(t)}$ between the previous residual iteration and the MIMO channels, a potential support set $\mathbf{P}$ which makes the correlation $\mathbf{u}^{(t)}$ largest will be selected from the predefined spatial constellation set.

(4) After updating the current support set $\mathbf{T}_{k}$, wrong indices will be removed and most likely indices will be selected according to the least squares.

(5) The normalization preprocessing allows different measurement matrices during the sensing and recovery process; the matrix during the sensing process is $\mathbf{H}^{\prime}$ in lines (6) (12) and the matrix during the recovery process is $\mathbf{H}$ in line (13). 
TABLE 1: Simulation parameters.

\begin{tabular}{lc}
\hline Parameters & Value \\
\hline $\begin{array}{l}\text { Transmit antenna number at BS } \\
\text { Transmit antenna number in } \\
\text { each user }\end{array}$ & 96 \\
Receive antenna number at BS & 1 \\
Modulate mode & 64 \\
Noise model & 16-QAM \\
& Gaussian/non-Gaussian \\
\end{tabular}

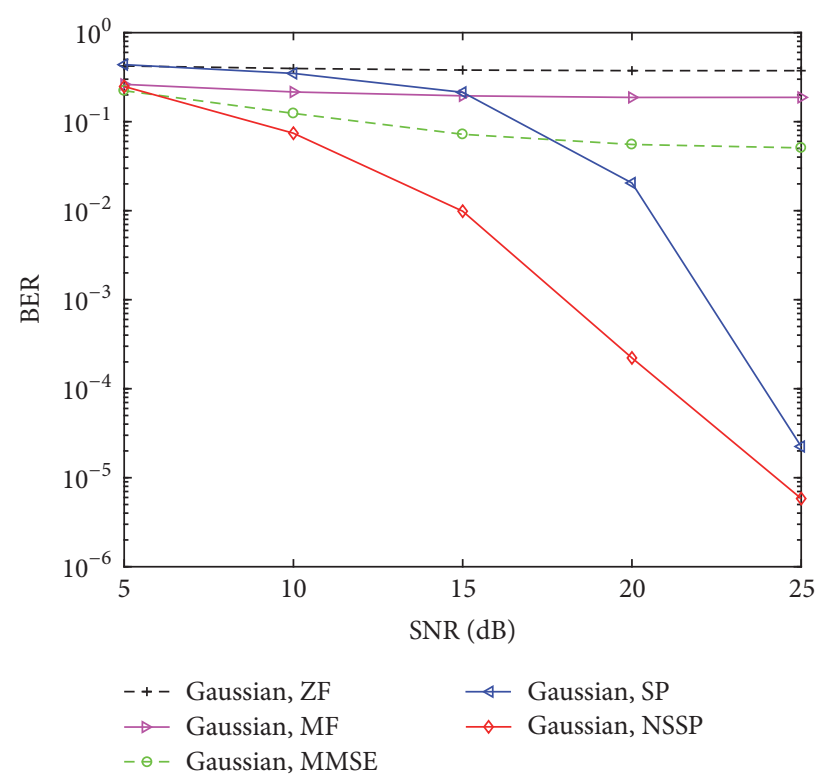

FIGURE 2: BER versus SNR performance of ZF, MF, MMSE, SP, and NSSP over uncorrelated Rayleigh-fading MIMO channels, where $K=24, N_{a}=24, N_{t}=96, N_{r}=64, G=1$, and 16QAM and Gaussian noise are considered.

(6) The parameter of output is the estimated signal $\widehat{\mathbf{x}}^{(t)}=$ $\left(\mathbf{H}_{T_{k}}^{(t)}\right)^{\dagger} \mathbf{y}^{(t)}$.

\section{Performance Analysis}

Here we present the bit error rate (BER) performance of the proposed algorithm and several signal detection algorithms. The ZF, MF, and MMSE detection is simulated for comparisons. And the SP algorithm is chosen as CS based signal detection for comparison. The parameters are listed in Table 1. $N_{t}$ is the number of transmit antennas, $N_{a}$ is the number of active antennas in each user, and $N_{r}$ is the number of receive antennas at the receiver. 16QAM signal constellation set is considered.

Figure 2 shows BER versus signal to noise ratio (SNR) performance of ZF, MF, MMSE, SP, and NSSP over uncorrelated Rayleigh-fading MIMO channels, where $K=24, N_{a}=24$, $N_{t}=96, N_{r}=64, G=1$, and 16QAM and Gaussian noise are considered. It can be observed that the proposed NSSP algorithm outperforms the conventional signal detectors since the normalization preprocessing is considered and the

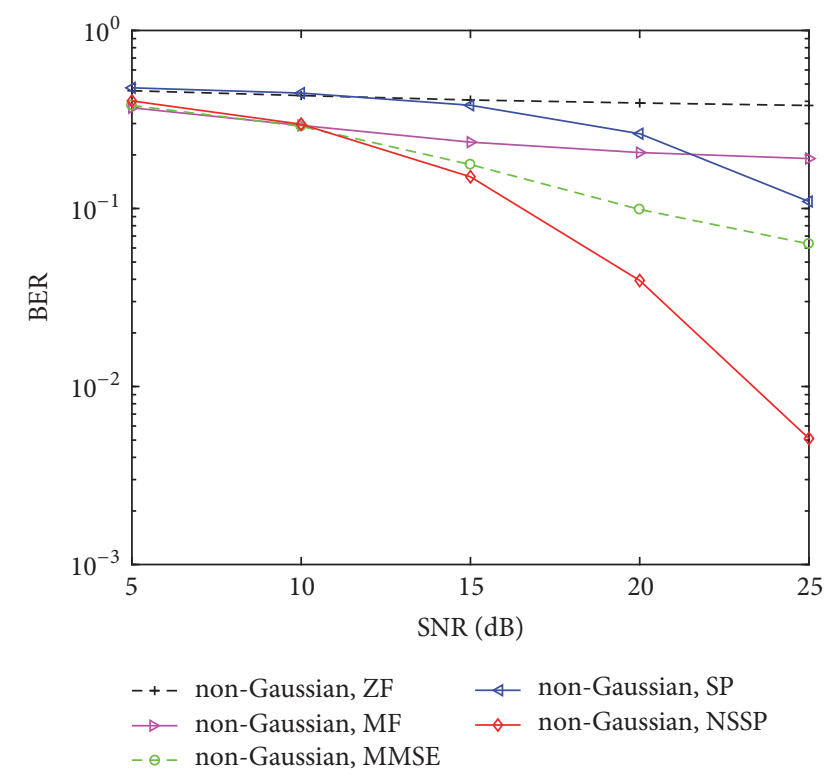

FIgURE 3: BER versus SNR performance of ZF, MF, MMSE, SP, and NSSP over uncorrelated Rayleigh-fading MIMO channels, where $K=24, N_{a}=24, N_{t}=96, N_{r}=64, G=1$, and 16QAM and non-Gaussian noise are considered.

structured sparsity of sparse signals is exploited. The performance of the NSSP algorithm and the SP algorithm is similar under the Gaussian noise.

Figure 3 shows BER versus SNR performance of ZF, MF, MMSE, SP, and NSSP over uncorrelated Rayleigh-fading MIMO channels, where $K=24, N_{a}=24, N_{t}=96, N_{r}=64$, $G=1$, and 16QAM and non-Gaussian noise are considered. It is obvious that the NSSP algorithm gives better performance than conventional signal detectors especially when the SNR is comparatively high. Moreover, The NSSP algorithm performs much better than the SP algorithm under the non-Gaussian noise.

Figures 4 and 5 shows BER versus SNR performance of the NSSP algorithm under Gaussian noise and non-Gaussian noise, respectively, with different numbers of transmit antennas and receive antennas. For example, NSSP- $64 * 48$ means there are 64 transmit antennas and 48 receive antennas in this simulation. It may be concluded that the NSSP algorithm has stable and reliable performance under both Gaussian and non-Gaussian noise. Moreover, it performs better when the numbers of transmit and receive antennas are larger.

\section{Conclusion}

This paper proposed a structured compressed sensing based signal detection for the SM-3D-MIMO system. Firstly, normalization preprocessing is considered before iteration process with the purpose of preventing the noise from being overamplified by the column vector with inadequately large elements. Secondly, the inherent sparsity of 3D-SM-MIMO signals is utilized in signal detection to reduce computation. Finally, simulation results have been provided to confirm the merits of the proposed methods in detection. 


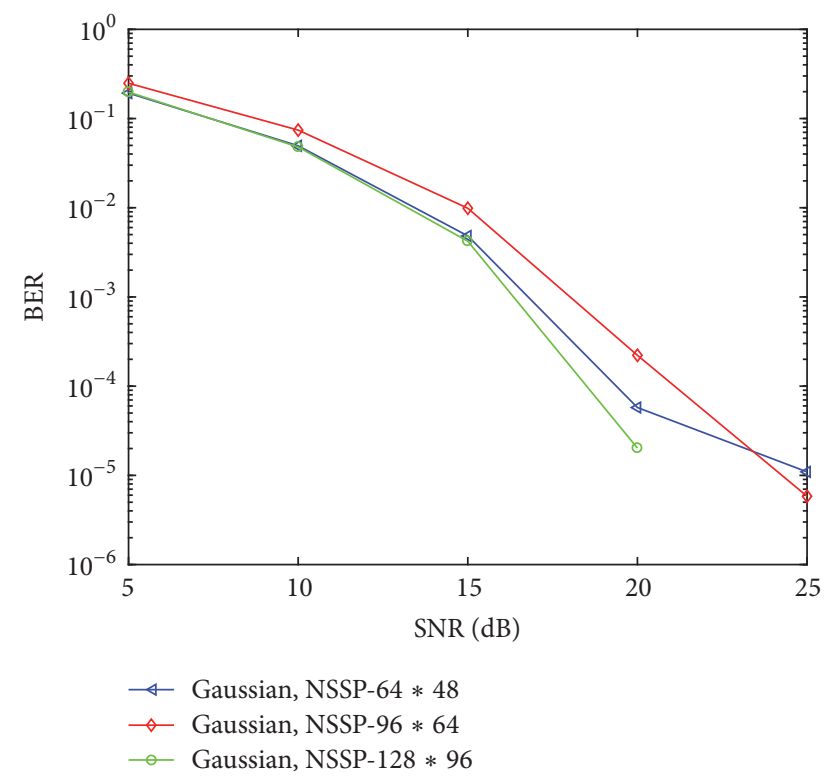

FIGURE 4: BER versus SNR performance of the NSSP algorithm under Gaussian noise with different numbers of transmit and receive antennas.

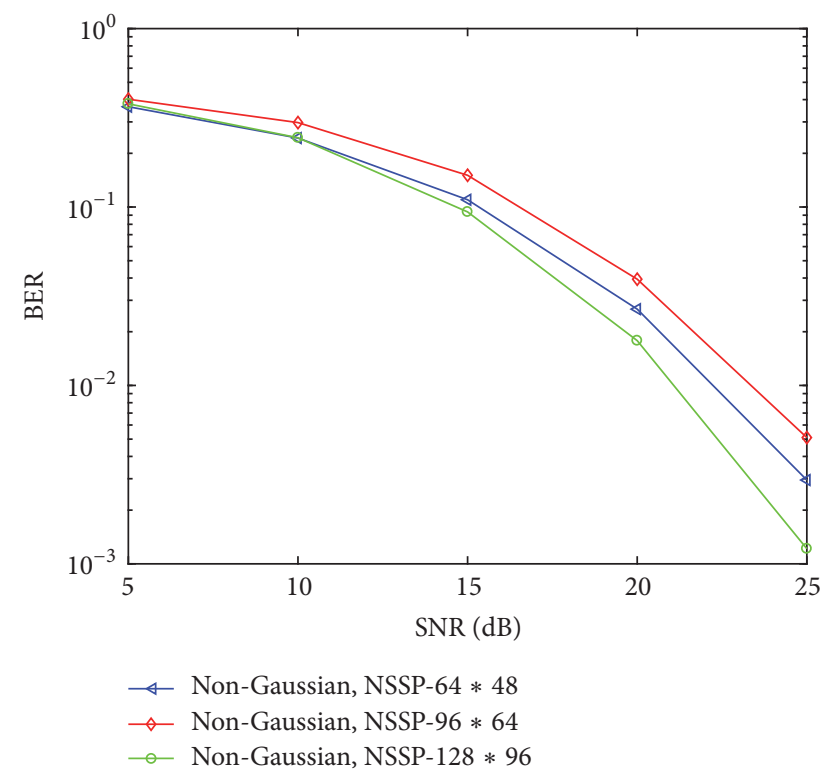

FIGURE 5: BER versus SNR performance of the NSSP algorithm under non-Gaussian noise with different numbers of transmit and receive antennas.

\section{Conflicts of Interest}

The authors declare no conflicts of interest.

\section{Acknowledgments}

This work was supported by National Natural Science Foundation of China (Grants nos. 61471200 and 61401069), Jiangsu Specially Appointed Professor Grant (RK002STP16001), Innovation and Entrepreneurship of Jiangsu High-level Talent Grant (CZ0010617002), high-level talent startup grant of Nanjing University of Posts and Telecommunications (XK0010915026), and "1311 Talent Plan" of Nanjing University of Posts and Telecommunications.

\section{References}

[1] R. Y. Mesleh, H. Haas, S. Sinanović, C. W. Ahn, and S. Yun, "Spatial modulation," IEEE Transactions on Vehicular Technology, vol. 57, no. 4, pp. 2228-2241, 2008.

[2] F. Fang, H. Zhang, J. Cheng, and V. C. M. Leung, "Energyefficient resource allocation for downlink non-orthogonal multiple access network," IEEE Transactions on Communications, vol. 64, no. 9, pp. 3722-3732, 2016.

[3] J. Zhang, Y. Zhang, Y. Yu, R. Xu, Q. Zheng, and P. Zhang, "3-D MIMO: how much does it meet our expectations observed from channel measurements?" IEEE Journal on Selected Areas in Communications, vol. 35, no. 8, pp. 1887-1903, 2017.

[4] X. Cheng, B. Yu, L. Yang et al., "Communicating in the real world: 3D MIMO," IEEE Wireless Communications Magazine, vol. 21, no. 4, pp. 136-144, 2014.

[5] M. Dong, K. Ota, and A. Liu, "RMER: reliable and energyefficient data collection for large-scale wireless sensor networks," IEEE Internet of Things Journal, vol. 3, no. 4, pp. 511-519, 2016.

[6] G. Auer, "3D MIMO-OFDM channel estimation," IEEE Transactions on Communications, vol. 60, no. 4, pp. 972-985, 2012.

[7] Z. Zhou, J. Gong, Y. He, and Y. Zhang, "Software defined machine-to-machine communication for smart energy management," IEEE Communications Magazine, vol. 55, no. 10, pp. 52-60, 2017.

[8] N. Zhao, F. R. Yu, and V. C. M. Leung, "Wireless energy harvesting in interference alignment networks," IEEE Communications Magazine, vol. 53, no. 6, pp. 72-78, 2015.

[9] P. Yang, M. Di Renzo, Y. Xiao, S. Li, and L. Hanzo, "Design guidelines for spatial modulation," IEEE Communications Surveys \& Tutorials, vol. 17, no. 1, pp. 6-26, 2015.

[10] M. D. Renzo, H. Haas, A. Ghrayeb, S. Sugiura, and L. Hanzo, "Spatial modulation for generalized MIMO: challenges, opportunities, and implementation," Proceedings of the IEEE, vol. 102, no. 1, pp. 56-103, 2014.

[11] Z. Xu, S. Sfar, and R. S. Blum, "Analysis of MIMO systems with receive antenna selection in spatially correlated Rayleigh fading channels," IEEE Transactions on Vehicular Technology, vol. 58, no. 1, pp. 251-262, 2009.

[12] J. Jeganathan, A. Ghrayeb, and L. Szczecinski, "Spatial modulation: optimal detection and performance analysis," IEEE Communications Letters, vol. 12, no. 8, pp. 545-547, 2008.

[13] M. D. Renzo, H. Haas, and P. M. Grant, "Spatial modulation for multiple-antenna wireless systems: a survey," IEEE Communications Magazine, vol. 49, no. 12, pp. 182-191, 2011.

[14] N. R. Naidoo, H. J. Xu, and T. Al-Mumit Quazi, "Spatial modulation: optimal detector asymptotic performance and multiplestage detection," IET Communications, vol. 5, no. 10, pp. 13681376, 2011.

[15] N. Zhao, F. R. Yu, and V. C. M. Leung, "Opportunistic communications in interference alignment networks with wireless power transfer," IEEE Wireless Communications Magazine, vol. 22, no. 1, pp. 88-95, 2015.

[16] J. Zheng, "Signal vector based list detection for spatial modulation," IEEE Wireless Communications Letters, vol. 1, no. 4, pp. 265-267, 2012. 
[17] C. B. Joao and S. N. Raimundo, "Low-complexity sphere decoding detector for generalized spatial modulation MIMO systems," IEEE Communications Letters, vol. 18, no. 6, pp. 949952, 2014

[18] F. Gao, T. Cui, and A. Nallanathan, "On channel estimation and optimal training design for amplify and forward relay networks," IEEE Transactions on Wireless Communications, vol. 7, no. 5, pp. 1907-1916, 2008.

[19] M. Lustig, D. L. Donoho, J. M. Santos, and J. M. Pauly, "Compressed sensing MRI," IEEE Signal Processing Magazine, vol. 25, no. 2, pp. 72-82, 2008.

[20] J. A. Tropp and A. C. Gilbert, "Signal recovery from random measurements via orthogonal matching pursuit," Institute of Electrical and Electronics Engineers Transactions on Information Theory, vol. 53, no. 12, pp. 4655-4666, 2007.

[21] A. Garcia-Rodriguez and C. Masouros, "Low-complexity compressive sensing detection for spatial modulation in large-scale multiple access channels," IEEE Transactions on Communications, vol. 63, no. 7, pp. 2565-2579, 2015.

[22] Z. Gao, L. Dai, C. Qi, C. Yuen, and Z. Wang, "Near-optimal signal detector based on structured compressive sensing for massive SM-MIMO," IEEE Transactions on Vehicular Technology, vol. 66, no. 2, pp. 1860-1865, 2017.

[23] W. Ren, G. Gui, and F. Li, "Performance evaluation of structured compressed sensing based signal detection in spatial modulation 3D MIMO systems," in Proceedings of the in International Conference on Advanced Hybrid Information Processing (ADHIP), pp. 1-9, Harbin, China, 2017.

[24] X. Wu, H. Claussen, M. Di Renzo, and H. Haas, "Channel estimation for spatial modulation," IEEE Transactions on Communications, vol. 62, no. 12, pp. 4362-4372, 2014.

[25] L. Tong and S. Perreau, "Multichannel blind identification: from subspace to maximum likelihood methods," Proceedings of the IEEE, vol. 86, no. 10, pp. 1951-1968, 1998.

[26] C.-M. Yu, S.-H. Hsieh, H.-W. Liang et al., "Compressed sensing detector design for space shift keying in MIMO systems," IEEE Communications Letters, vol. 16, no. 10, pp. 1556-1559, 2012.

[27] D. L. Donoho, "Compressed sensing," Institute of Electrical and Electronics Engineers Transactions on Information Theory, vol. 52, no. 4, pp. 1289-1306, 2006.

[28] E. J. Candès and M. B. Wakin, "An introduction to compressive sampling: a sensing/sampling paradigm that goes against the common knowledge in data acquisition," IEEE Signal Processing Magazine, vol. 25, no. 2, pp. 21-30, 2008.

[29] S. Foucart, "Hard thresholding pursuit: an algorithm for compressive sensing," SIAM Journal on Numerical Analysis, vol. 49, no. 6, pp. 2543-2563, 2011.

[30] M. F. Duarte and Y. C. Eldar, "Structured compressed sensing: from theory to applications," IEEE Transactions on Signal Processing, vol. 59, no. 9, pp. 4053-4085, 2011. 


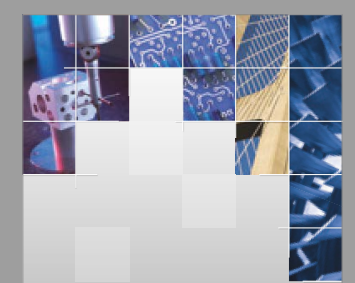

\section{Enfincering}
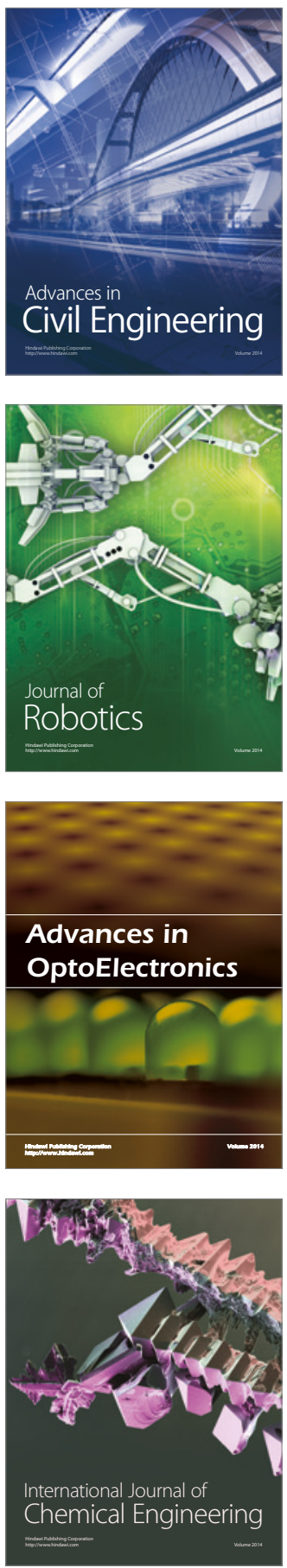

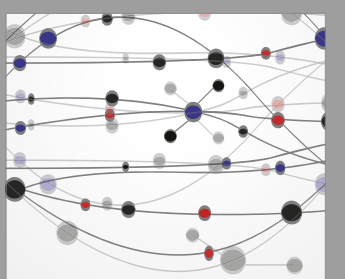

The Scientific World Journal

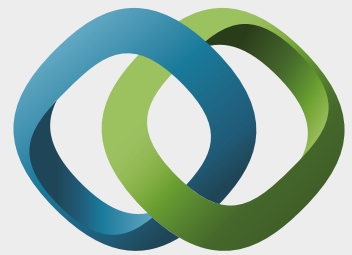

\section{Hindawi}

Submit your manuscripts at

https://www.hindawi.com
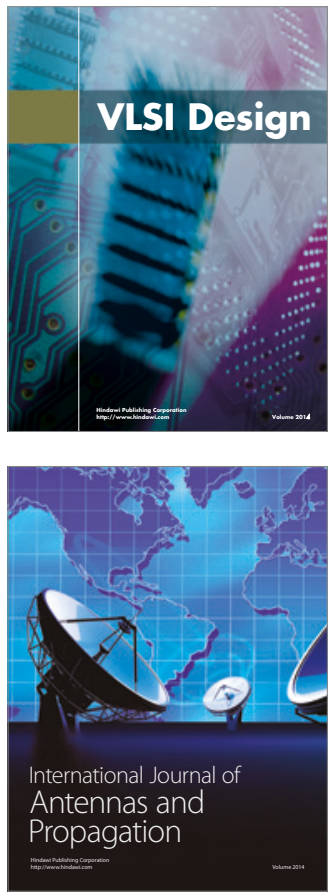

\section{Rotating}

Machinery
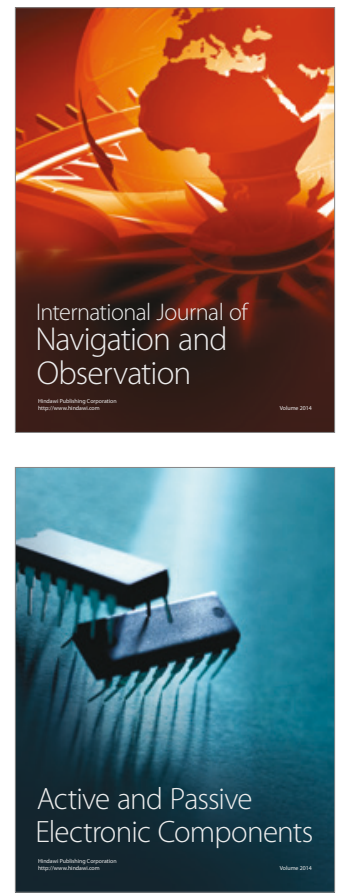
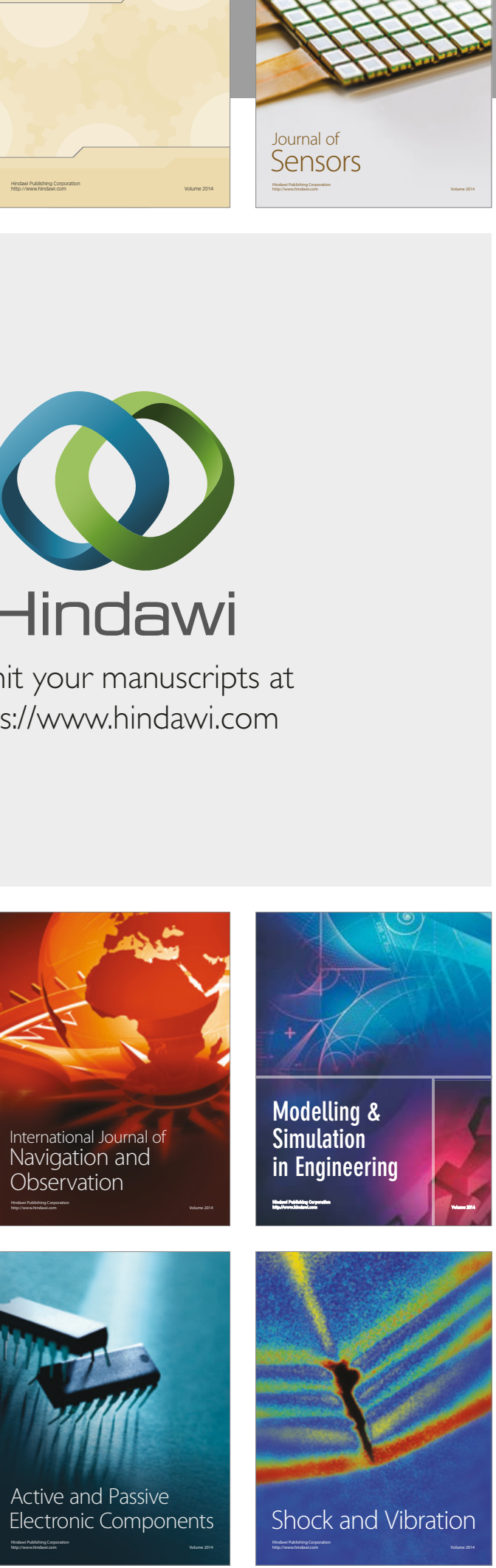
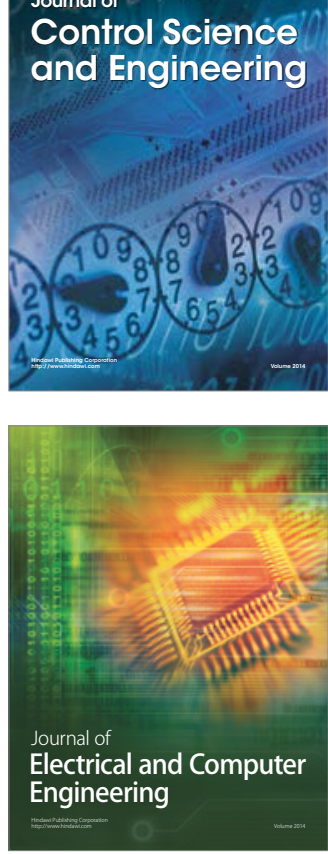

Distributed

Journal of

Control Science

and Engineering
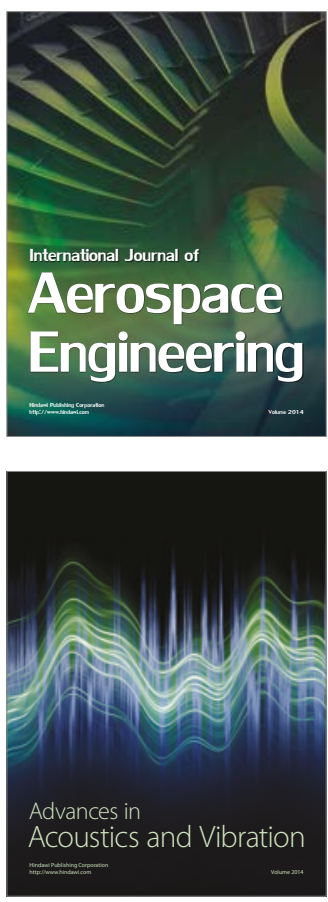

Sensor Networks 